\title{
A Wireless Sensor Network based Market Parking Scheme
}

\author{
Ajobiewe, N. Damilola \\ Kwame Nkrumah University of Science and Technology. \\ Dept. of Electrical /Electronics Engineering, \\ Kumasi, Ghana.
}

\author{
Willie, K. Ofosu \\ Penn State University. \\ Wilkes Barre. \\ Lehman, P.A 18627
}

\author{
Oyibo, A. Michael \\ Kwame Nkrumah University of Science and Technology. \\ Dept. of Electrical /Electronics Engineering, \\ Kumasi, Ghana.
}

\begin{abstract}
Parking in major cities, especially around a market vicinity is not only limited but costly. Innovative parking systems to curb this phenomenon is required. This paper proposes a novel and strategic approach using wireless sensor networks (WSNs) as a solution. The WSNs consisting of sensor nodes are known for offering detection, sensing and communication services. The market parking scheme serves as smart parking service, for monitoring the state of the market parking garage. To function, each slot within the parking space is fit with a magnetic sensor node. The parking process is modelled as an Entry-Exit stochastic process. Vehicles identified as they approach using the Licence Plate Recognition (LPR) program. Post identification, Low-Energy Adaptive Clustering Hierarchy $(\mathrm{LEACH})$ routing protocol of the WSNs is used to route the information concerning the parking garage to the base station. The Performance and evaluation of this proposed innovatory system, proves its efficiency.
\end{abstract}

\section{General Terms:}

Smart Parking, Wireless Sensor Networks

\section{Keywords:}

Wireless Sensor Networks; Vehicle Detection; Wireless Communication; Smart Parking

\section{INTRODUCTION}

Parking of vehicles in market environment has become critical as businesses depend largely on the number of patrons. The size of a parking lot depends largely on the number of vehicles it is proposed to accommodate and the size of the kind of business operated there [1].

Businesses with a great deal of customers would require a large parking space/garage, unlike the small and medium scale business with lesser patronage. On the average, a $30 \%$ estimate of the approximated number of vehicles driving on the roads in the down- town area of major cities are said to be cruising around in search of a parking spot [2].

And it approximately takes an average of not less than 7.8 minutes to find one. The problem of traffic congestion on market days around market environments has become frequent hot button subject matter in the news ${ }^{1}$ and this problem requires further research for a solution.

The basic idea of this paper is to present a system that optimizes parking and reduce traffic congestion in market environments using wireless sensor networks. In the next Section, the factors influencing the causes of parking issues and traffic congestion are described. Section 3 highlights related works done, and Section 4 describes the system model used. Section 5 shows, the flowchart iterative parking guidance and in Section 6, simulation results are presented. In section 7, the conclusions drawn.

\section{FACTORS INFLUENCING THE CAUSE OF PARKING ISSUES}

Inaccessibility to parking facilities can adversely affect local businesses and can affect the quality of life of residents. Common complaints amongst motorists includes; someone is either parked in their allocated space or someone is parking too long or too frequently in a visitor car park. Parking in a place that is not designated for parking can cause traffic jam [3]|[4].

Commonly identified parking problems that typically occur in a market environment are;

-Inadequate Information for Motorists- Poor information for motorists on the parking availability and price. Provision of information on availability of parking space and price using signs, brochures and maps, websites, and parking information can be incorporated into general marketing materials. This will in turn reduce motorist delay and frustration, and increase user satisfaction. Also improving the use of information could be a solution to this challenge.

\footnotetext{
${ }^{1}$ http://www.citydata.com/worldcities/Lagos.html
} 
- Spill Over Problems- Restrictions, reductions in parking supply, and pricing can cause parking problems in nearby areas as motorists seek additional or cheaper parking. Dealing with the problems of spillover, can reduce problems and avoid public resistance to other parking management programs.

- Insufficient Parking at Event Site. Event centres with inadequate parking facilities on special event days can actually disrupt free traffic flow and require crowd management. However each event can bring forth its own unique transportation issues.

\section{RELATED WORKS}

At present, car parking systems use various techniques in vehicle detection and information acquisition. Many algorithms have also been proposed for monitoring vehicles in motion. Similarity Based Vehicle Detection (SBVD) algorithm to detect vehicles with low SNR conditions by estimating similarity and difference between on-road signals, and a referential signal have been proposed [5]. Smart parking systems utilizing Licence Plate Recognition (LPR), was developed to distinguish vehicles using the contents, character and colours on their licence plates. The LPR system consists of components such as image acquisition, licence plate extraction, segmentation, and recognition of individual characters. This system is most useful as smart parking, highway electronic toll collection, and traffic monitoring systems to mention a few. However LPR systems could sometimes be problematic due to low resolution of ana$\log$ video signal, and different licence plate configuration from the suitable computer based vision recognition [6, 7, 8].

Radio Frequency Identification (RFID) is an automatic identification method, which recalls remote information saved with RFID transponders or tags used in smart parking system [9]. Driving in and out of the parking-lots is controlled using RFID readers, labels, and barriers [10]. Motorists will not have to make any payments at each check-out and as a result, a faster traffic flow will be possible. The barrier is lifted only after the vehicle is verified. Remarkably a remote/ virtual database is maintained to continue the process when the internet is cut off or unavailable. The use of RFID technology provides security to the parking management system. However the system is vulnerable when more than one tag responds at the same time [11].

\section{SYSTEM MODEL}

In deploying the wireless sensor network based market parking scheme, the vehicle is detected by the sensor nodes. The vehicle detection process is as follows;

- magnetic signals from the vehicle is first detected

-a pre-processing signal stage

-fluctuation detection

—stability disturbance detection stage.

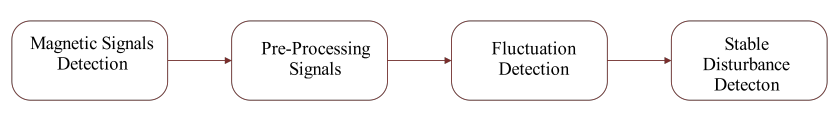

Fig. 1. Vehicle Detection Characteristics.

The figure 1 shows a block diagram of the vehicle detection process.

Magnetic Signal Detection: A significant volume of ferrous metals in the chassis and engine of the vehicle interferes with the earth's field. The system uses a magnetometer, which is able to sense the earth's magnetic field which is below 1 gauss. The earth's field is said to be uniform over a wide area in the scale of kilometres. The presence of the magnetic signature of the vehicle can be identified over the earth's magnetic field. The magnetic detecting sensors are buried in the ground. The oscillations and frequency produced in the loop of the detector depends on the inductance of the loop. The detector senses this change in the earth's magnetic field caused by the vehicle, and gives an output [12].

Pre-Processing of Signal: This occurs after the disturbance in the earth magnetic field as a result of the passing vehicle. The change in the earth's magnetic field is a key factor in the detection. Unfortunately the amplitude and direction of the magnetic field is low. The pre-processing signal unit consists of a communication unit and a calibration information generation unit. Information about the movement of a reference vehicle from a central management centre is received by the communication unit at the same time as the sensed information of the passing vehicle [13]. The calibration unit fine-tunes the information received from the communication unit using the information about the movement of the reference vehicle.

Using digitalized measurements of three-axis sensor outputs after amplification, the vector magnitude would be;

$$
A(i)=\sqrt{X_{i}^{2}+Y_{i}^{2}+Z_{i}^{2}}
$$

The signals are passed through a smoothing filter to obtain a running average as follows:

$$
P(i)= \begin{cases}\frac{A(i)+A(i-1)+\ldots+A(1)}{i} & i \geq L . \\ \frac{A(i)+A(i-1)+\ldots+A(i-L+1)}{L} & i<L,\end{cases}
$$

where $A(i)$ is the vector magnitude and $L$ is the running average of the buffer size.

\section{FLOWCHART FOR ITERATIVE PARKING GUIDANCE}

The flow chart illustrates the parking model for a typical parking garage to ensure the problem of traffic is reduced in and around the market places or event centres. In Fig 2 as the motorist approaches the entry point, the vehicle proceeds to MPs1, where it is verified and evaluated. It continues to MPs2, where the vehicle class is ascertained whether the motorist is a private user or a commercial user. It goes forward to MPs3 if and only if the motorist is a private vehicle owner, checks for availability of free space and parks. It terminates at MPs8. Else it moves to MPs4 to determine the route to the commercial vehicle park, checks for availability and parks. In the scenario where no parking space is available, both situations continues to a waiting stage at MPs7, and they are all terminated at the exit point MPs9.

The Fig 3 illustrates what happens when the sensor detects a vehicle.

As the vehicle drives into the parking lot and parks, the sensor node being idle goes to sleep as there is no activity taking place. It therefore remains in the parking state until the driver is leaving, when the movement causes a change or fluctuation in the earth's magnetic field signal. The spot goes back to the vacant state and the sensor becomes idle. In the next section, a simulation of the case scenario is presented. Interpretation of the results obtained from the analysis, which is based on the approach described above is also presented. 


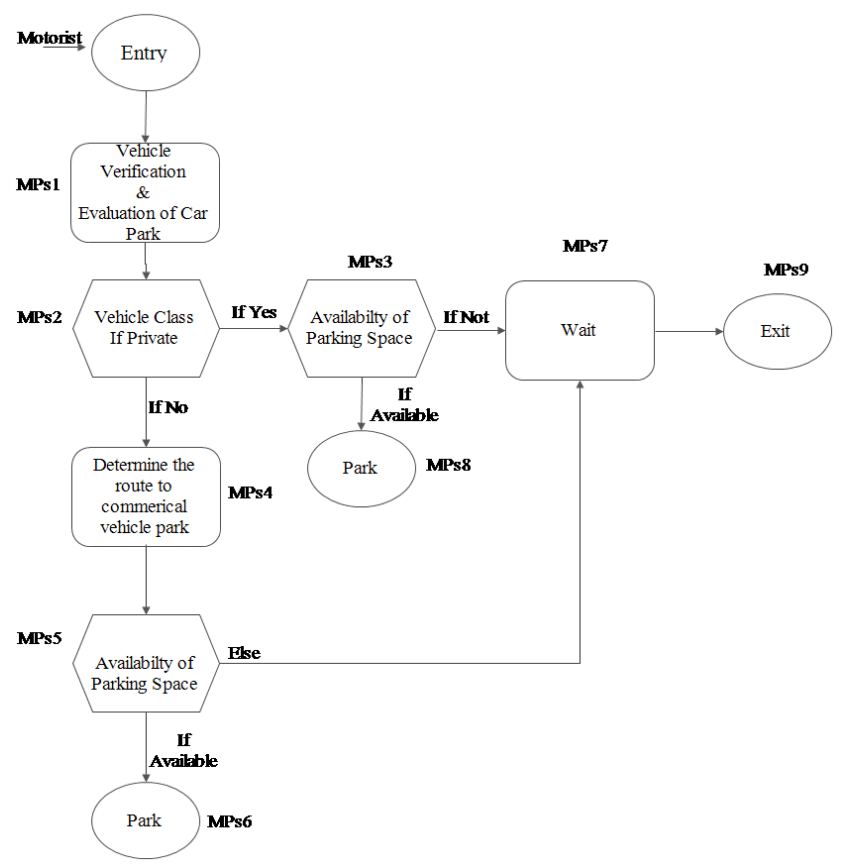

Fig. 2. Flowchart for Parking Guidance.

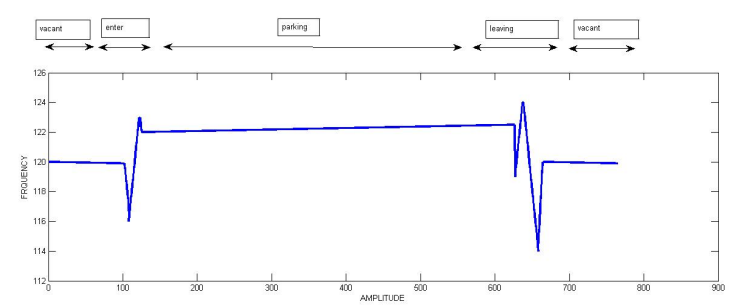

Fig. 3. Detection Activity of the Sensor Node.

\section{SIMULATION RESULTS}

The simulations in this work are executed using MATLAB (version R2012a). Through simulation,the sensor nodes are employed for vehicle detection and are evaluated for availability of parking space. The Radio Interferometric Positioning System (RIPS) of the localization system is applied after cluster formation within the sensor nodes have taken place, then the LEACH protocol is used to transmit the data to the administrator. The Hilbert transform is used to derive analytic representation of a signal which forms the basis of this simulation. Each figure consists of both simulation and analytical results. The Fig 4 shows a distinctive vehicle raw signal waveform. As stated above the sensors that are buried in the parking lots are magnetic sensors, and vehicles also possess a certain amount of ferrous components that are detected by the magnetic sensor. The transmitted signals are therefore signals sensed by the node as a vehicle approaches within a few meters. This shows a distinctive vehicle's raw signal waveform. The table above shows the list of parameters used for the simulation.

There can be a situation in which, some other object that is not ve-

\begin{tabular}{||ll||}
\hline Simulation Parameters & Value \\
\hline \hline Total Number of nodes & 1000 \\
\hline Number of Frames & 10 \\
\hline Coverage area & $20-200$ meters \\
\hline Monitoring area & $100 \mathrm{~m} \mathrm{x} \mathrm{100m}$ \\
\hline Node Initial Energy & $0.5 \mathrm{j}$ \\
\hline \multicolumn{2}{|l||}{ Parameters used for Simulation. } \\
\hline
\end{tabular}

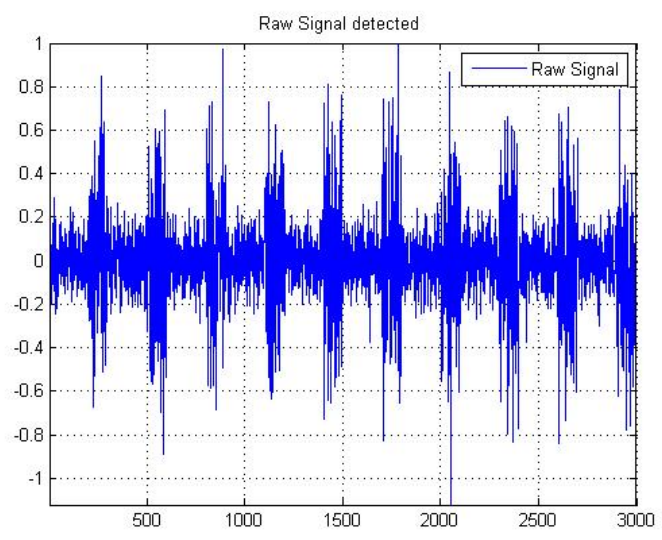

Fig. 4. Raw Signal Detected from Vehicle.

hicular possess similar ferrous component as the vehicle. The nodes could sense signals from these objects just as well.

Fig 5 shows signals received from non-vehicular objects. The de-

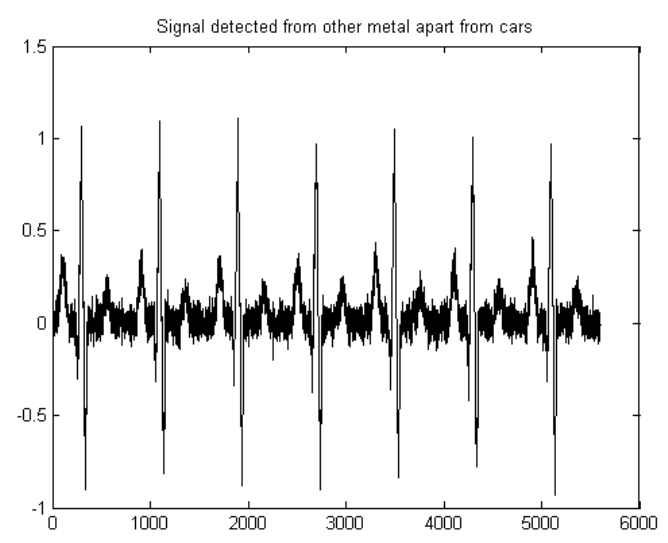

Fig. 5. Detected Signals From Non-Vehicle.

tection outcome whose value denotes the coordinate axis of the sensor nodes, where value " 0 " indicates the parking is "vacant," and "1" stands for "occupied", are shown in Fig 6 .

Fig 6 shows the occupancy of the parking space at intervals on daily bases. The figure also shows, signals from both vehicle and non-vehicle objects. The adaptive threshold and the activity of the vehicle are also shown.

After detection, the system utilizes the LEACH protocol to route the information to the base station. And the information about availability would be displayed on an information board which could be a LED display board. The LEACH being a standard, robust and 
Raw Signal and detected Onsets of activity

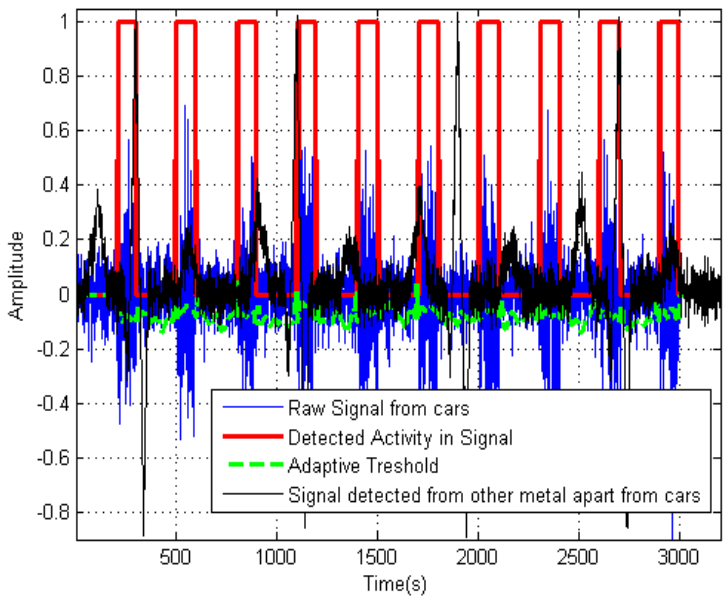

Fig. 6. Detect of Onset Activity.

widely spread hierarchical routing algorithm for sensor networks is adapted for this work. Also the LEACH is appropriate, in case of low consumption of energy, and it can sustain and improve the lifespan of the WSNs [14, 15].

\section{CONCLUSION}

In this paper, a market parking system is presented using wireless sensor networks. It concentrates on optimizing the parking system of market environments thereby curtailing traffic congestions.

The motorist is able to situate a parking spot easily and in so doing saves the tension of time wasting, frustration and environmental pollution.

Results from simulation prove the proposed system utilizes parking space and reduces time taken to find parking spot. Future work includes extended simulations on the proposal.

\section{REFERENCES}

[1] D. C. Shoup. The high cost of free parking. Journal of Planning Education and Research, 17(1):3-20, 1997.

[2] Y. Geng and C. G. Cassandras. A new smart parking system based on optimal resource allocation and reservations. In Intelligent Transportation Systems (ITSC), 2011 14th International IEEE Conference on, pages 979-984. IEEE, 2011.

[3] D. N. Ajobiewe. A market parking system using wireless sensor networks. Master's thesis, Kwame Nkrumah university of Science and Technology, Kumasi, Ghana., 2014.

[4] Banerjee \& Associates. An overview of common parking issues, parking management options, and creative solutions. Technical report, City of Pasadena; Department of Transportation, 2003.

[5] J. Yick, B. Mukherjee, and D. Ghosal. Wireless sensor network survey. Computer networks, 52(12):2292-2330, 2008.

[6] W. Zhou, H. Li, Y. Lu, and Q. Tian. Principal visual word discovery for automatic license plate detection. Image Processing, IEEE Transactions on, 21(9):4269-4279, 2012.

[7] M. Lalimi and S. Ghofrani. An efficient method for vehicle license plate detection in complex scenes. Circuits and Systems, 2:320, 2011.
[8] M. A. Lalimi, S. Ghofrani, and D. McLernon. A vehicle license plate detection method using region and edge based methods. Computers \& Electrical Engineering, 39(3):834845, 2013.

[9] C. Delaunay, E. Sahin, and Y. Dallery. A literature review on investigations dealing with inventory management with data inaccuracies. In RFID Eurasia, 2007 1st Annual, pages 1-7. IEEE, 2007.

[10] A. K. M. Masum, F. Bhuiyan, and K. G. Azam. Rfid applications: Prospects and challenges in bangladesh. Journal of Information Security, 4(2), 2013.

[11] R. Ranjini and D. Manivannan. A comparative review on car parking technologies. International Journal of Engineering \& Technology (0975-4024), 5(2), 2013.

[12] J. Ding, S. Y. Cheung, C. Tan, and P. Varaiya. Vehicle detection by sensor network nodes. 2004.

[13] Z. Zhang, X. Li, H. Yuan, and F. Yu. A street parking system using wireless sensor networks. International Journal of Distributed Sensor Networks, 2013:10, 2013.

[14] Y. Li, N. Yu, W. Zhang, W. Zhao, X. You, and M. Daneshmand. Enhancing the performance of leach protocol in wireless sensor networks. In Computer Communications Workshops (INFOCOM WKSHPS), 2011 IEEE Conference on, pages 223-228. IEEE, 2011.

[15] M. Abdo-Saif and M. N. Shanmukha Swamy. A novel algorithm to select cluster heads with highest and balanced energy in wireless sensor networks. International Journal of Computer Applications, 54(4):19-24, September 2012. Published by Foundation of Computer Science, New York, USA. 